\title{
Performance Evaluation of a $4 \times$ 20-Gbps OFDM-Based FSO Link Incorporating Hybrid W-MDM Techniques
}

\author{
Mehtab Singh ${ }^{1,2}$, Saleh Chebaane ${ }^{3,4}$, Sana Ben Khalifa ${ }^{5,6 *}$, Amit Grover $^{7}$, Sanjeev Dewra ${ }^{7}$ \\ and Mohit Angurala ${ }^{8}$
}

${ }^{1}$ Department of Electronics and Communication Engineering, SIET Amritsar (IKG-PTU, Kapurthala), Amritsar, India, ${ }^{2}$ Department of Electronics and Communication Engineering, Guru Nanak Dev University, Regional Campus, Jalandhar, India, ${ }^{3}$ Department of Physics, College of Science, University of Ha'il, Ha'il, Saudi Arabia, ${ }^{4}$ Laboratoire d'électronique et micro-électronique (LAB-IT06) Faculté des Sciences de Monastir, Monastir, Tunisia, ${ }^{5}$ Department of Physics, College of Science and Arts, Qassim University, Ar Rass, Saudi Arabia, ' ${ }^{2}$ aboratory of Energy and Materials (LabEM), ESSTHS, University of Sousse, H. Sousse, Tunisia, ${ }^{7}$ Department of Electronics and Communication Engineering, Shaheed Bhagat Singh State University, Ferozepur, India, ${ }^{8}$ Department of Computer Science and Engineering, Chitkara University, Rajpura, India

OPEN ACCESS

Edited by:

Santosh Kumar

Liaocheng University, China

Reviewed by:

Sushank Chaudhary,

Quanzhou Institute of Equipment manufacturing, (CAS), China

Ayyanar Natesan,

Ton Duc Thang University, Vietnam

${ }^{*}$ Correspondence:

Sana Ben Khalifa

s.benkhalifa@qu.edu.sa

Specialty section: This article was submitted to

Optics and Photonics,

a section of the journal

Frontiers in Physics

Received: 24 July 2021

Accepted: 31 July 2021

Published: 20 September 2021

Citation:

Singh M, Chebaane S, Ben Khalifa S,

Grover A, Dewra $S$ and Angurala $M$ (2021) Performance Evaluation of a $4 \times$

20-Gbps OFDM-Based FSO Link

Incorporating Hybrid W-

MDM Techniques.

Front. Phys. 9:746779.

doi: $10.3389 /$ fphy.2021.746779
Free space optics (FSO) has been recognized as a crucial technique to meet the highbandwidth requirements in future wireless information transmission links. It provides a feasible solution to the last-mile bottleneck problem due to its merits that include highspeed data transportation and secure and low-latency networks. Due to these merits, FSO is a reliable technology for future health-care and biomedical services like the transmission of biomedical sensor signals. But the main limiting factor in the data transmission employing FSO links is adverse atmospheric weather conditions. This research work reports the designing and simulative evaluation of the performance of a high-speed orthogonal frequency division multiplexing-based free space optics link by incorporating wavelength division multiplexing of two independent frequency channels (193.1 THz and 193.2 THz) along with mode division multiplexing of distinct spatial laser Hermite-Gaussian modes (HG01 and HG03). Four independent 20-Gbps quadrature amplitude-modulated data signals are transported simultaneously under different atmospheric weather conditions using the proposed link. Also, the link performance has been investigated for an increasing beam divergence angle.

Keywords: FSO, WDM, MDM, HG modes, atmospheric weather conditions, beam divergence, biomedical services

\section{INTRODUCTION}

Recent years have seen a significant increase in network traffic due to growth in the use of multimedia applications consuming high channel bandwidth like video conferencing, fast Internet, and live streaming. This has challenged the limited and congested radio frequency (RF) spectrum-based conventional wireless transmission systems [1]. Free space optics (FSO) is considered a promising solution to meet the high capacity and large transmission rate demand of the users. Optically modulated carrier signals are used to carry data signals over the free space medium between tightly aligned transmitter and receiver units. FSO technology has numerous merits, such as quick and easy installation, high channel bandwidth, immunity to electromagnetic interference, a high-speed 


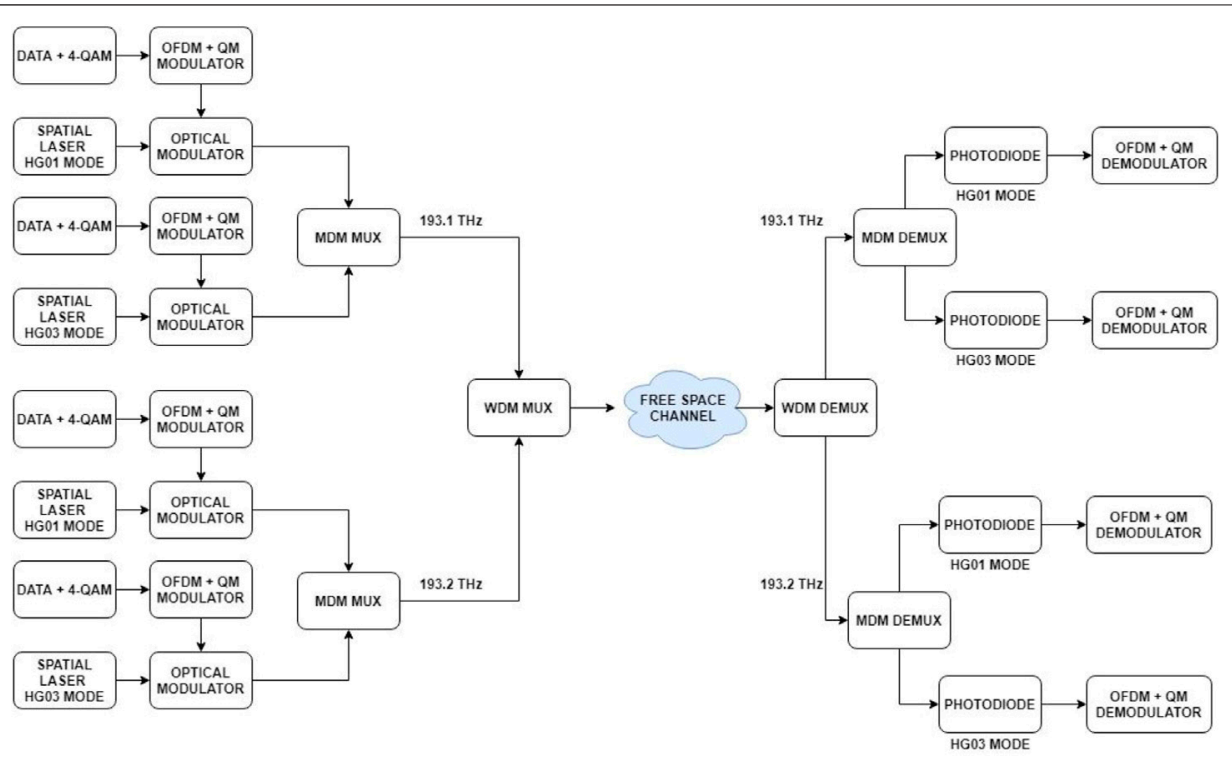

FIGURE 1 | Schematic design of the proposed link.

network, secure data transmission, and license-free spectrum availability [2-5]. Orthogonal frequency division multiplexing (OFDM) is a subset of multi-carrier modulation techniques using which a high-bit rate signal is transported over several low-speed subcarriers, which are spaced closely in the frequency domain and are orthogonal to each other, thus eliminating the intercarrier interference $[6,7]$. By incorporating OFDM technology with FSO links, highly reliable long-reach data transmission links can be realized. Here, the authors in references [8-10] report the design and performance investigation of the OFDM-based FSO terrestrial link under the effect of different atmospheric conditions. To increase the data-carrying capacity of the link, wavelength division multiplexing (WDM) can be used which transmits multiple information signals at the same time over the same medium using different wavelengths [11-14].

Mode division multiplexing (MDM) is an important and evolving transmission technique that capitalizes on different spatial modes of a single laser beam to transport independent data signals over the same channel. The authors in references [15-17] report optical signal-processing techniques to generate and de-multiplex different laser modes. The application of a spatial light modulator to multiplex and de-multiplex optical spatial laser beams has been reported in references [18, 19]. Y. Jung et al. proposed the application of dual-fused optical fiber for MDM transmission applications in reference [20]. A. Amphawan et al. report the application of a photonic crystal fiber with a single core to generate different linear polarized (LP) modes. In recent years, the incorporation of MDM in optical fiber links has been extensively investigated to realize high-speed transmission [21]. A. Juarez et al. reported an MDM system capable of realizing high-speed data transmission in multi-mode fiber (MMF) links using linear polarized (LP) modes [22]. The authors reported feasible transportation of 120 -Gbps data with $3 \mathrm{GHz}-\mathrm{km}$ bandwidth-length product over an MMF link of $50 \mathrm{~km}$ length

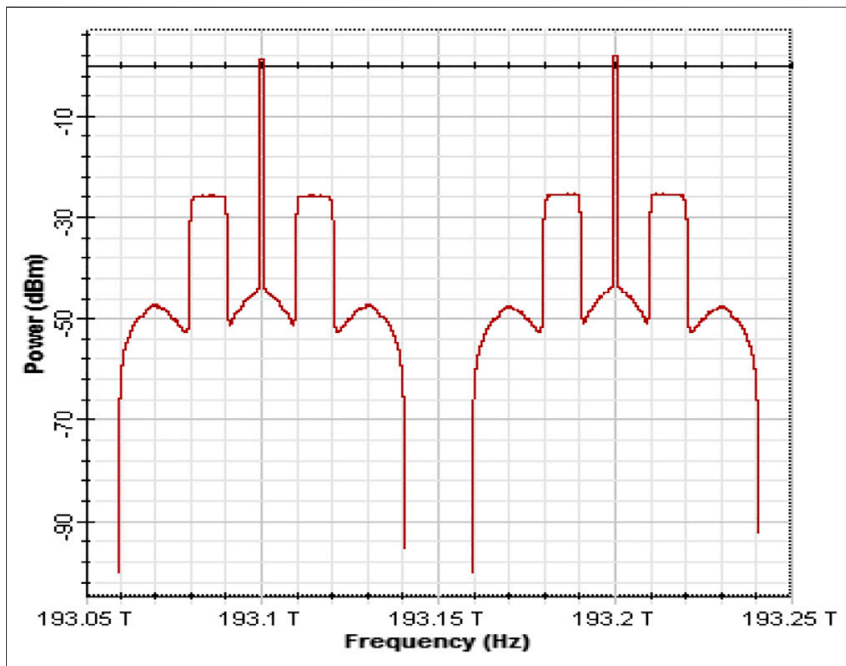

FIGURE 2 | Optical spectrum of the transmitted signal.

using a multi-mode erbium-doped fiber amplifier (EDFA) at the receiver unit. T. Kodama et al. reported a novel hybrid all-optical MDM code division multiplexed system to realize future generation optical access networks [23]. The authors experimentally reported feasible transmission of 2 LP modes $\times$ 4 phase-shift-keyed optical codes $\times 10$-Gbps on-off-keyed data streams over a $42 \mathrm{~km}$ fiber length using a single-mode and a multi-mode fiber without the application of dispersion compensation. T. Masunda et al. proposed hybrid MDM and WDM architecture to realize high-speed MMF interconnects[24]. Six independent vertical-cavity surface-emitting laser diodes are used, where each wavelength generates three distinct Laguerre-Gaussian (LG) modes to realize 18 parallel channel transmissions. The authors report feasible $60 \mathrm{Gbps}$ transmission 

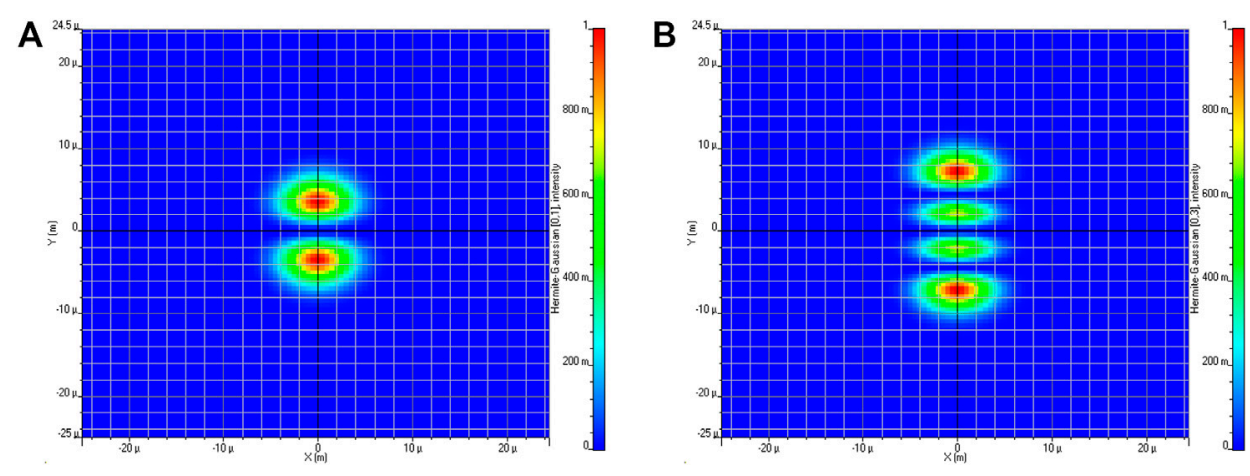

FIGURE 3 | Mode intensity profile of (A) HG01 and (B) HG03.
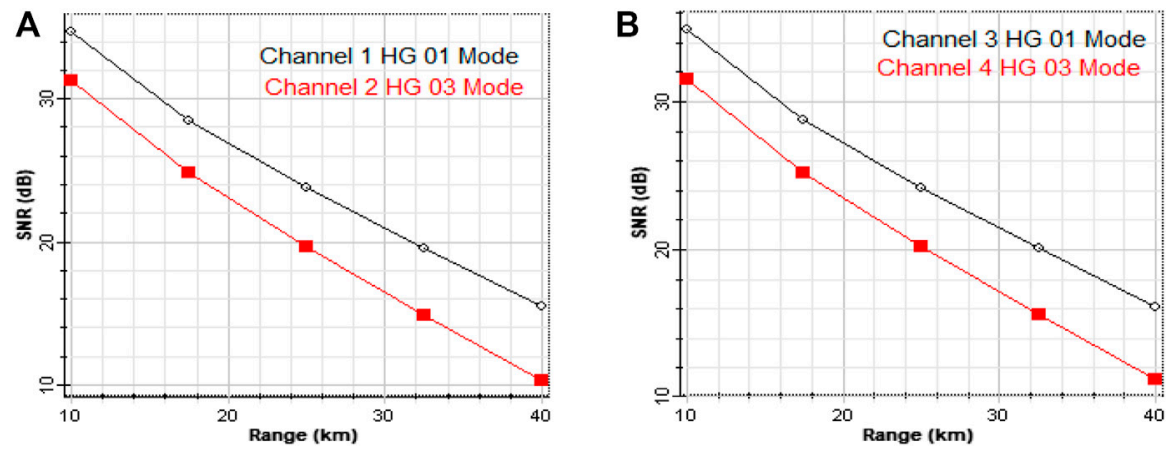

FIGURE 4 | SNR for (A) 1931. THz channel and (B) 193.2 THz channel under clear weather.

over a $2.5-\mathrm{km}$ MMF link by using a novel tap configuration in a feed-forward equalizer to mitigate the effects of inter-mode coupling.

R. Murad et al. reported a high-capacity MDM system using hybrid modes for high-capacity optical interconnects in data centers [25]. The authors reported feasible transportation of 44-Gbps data using two helical-phased ring modes over a 1,550.12-nm channel and two radially offset Hermite-Gaussian (HG) modes over a 1,551.72-nm channel along an MMF of $1,500 \mathrm{~m}$ range with an acceptable bit error rate of the system. E. Hamed et al. reported the performance comparison of three different types of optical fibers, that is, a step-index few-mode fiber (FMF), graded-index FMF, and transversal index-FMF in a spectral-efficient MDM system [26]. Three distinct LP modes, where each mode carries 10 -Gbps quadrature amplitudemodulated (QAM) data signals are transmitted over all the three optical fiber types. The authors reported that transversal index-FMF performs the best and demonstrated a feasible $500 \mathrm{~km}$ transmission of 30-Gbps QAM data with good performance. Z. Feng et al. reported an ultra-high channel capacity optical access network based on hybridization of MDM and WDM technologies with advanced modulation formats [27]. The incorporation of the 200-Gbps polarization division multiplexed (PDM)-16-levelQAM-OFDM data signal has been proposed in the system.
The authors reported feasible data transmission of 4 wavelength channels $\times 6$ spatial modes $\times 200$ Gbps QAM data signals along a $37-\mathrm{km}$ MMF with seven cores with acceptable performance.

The application of the orbital angular momentum (OAM) dimension of the optical signal to carry different independent information channels for realizing high-speed optical networks has been reported by many research groups in the last few years. The design of a hollow-core optical fiber capable of transporting 16 distinct OAM modes to realize highcapacity long-range MDM transmission has been reported by C. Brunet et al. in reference [28]. X. Zhang et al. reported the fabrication of a circular photonic crystal fiber capable of supporting 14 distinct OAM modes with low confinement losses and low nonlinear coefficients [29]. Also, the authors reported the fabrication of a multi-mode EDFA based on the circular photonic crystal fiber capable of reliably amplifying all 14 modes with $20 \mathrm{~dB}$ gain. The designing of a novel photonic crystal fiber, capable of supporting 26 OAM modes with low confinement loss, low nonlinear coefficient, and high bandwidth for long-haul spectrum-efficient MDM transmission in future optical access networks, has been reported by $\mathrm{M}$. Hassan et al. in reference [30]. K. Ingerslev et al. reported feasible 

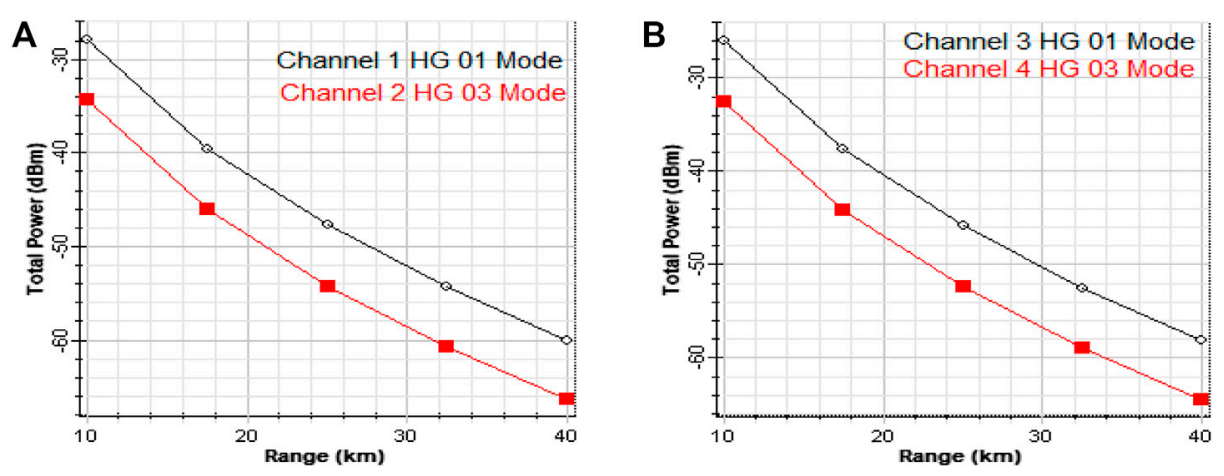

FIGURE 5 | Signal power for (A) 1931. THz channel and (B) 193.2 THz channel under clear weather.

transportation of 12 OAM modes, where each mode carries 10 Gbaud quadrature phase-shift-keyed (QPSK) signals over a 1.2-km MMF link with good performance [31]. Further, the authors have demonstrated ultrahigh capacity reliable transmission by using 60 independent wavelength channels with a channel spacing of $25 \mathrm{GHz}$. A. Tatarczak et al. reported an experimental demonstration of feasible transportation of three distinct OAM modes, where each mode transported a 10Gbps on-off-keyed signal over a 400-m MMF link for shortreach links and high-capacity data centers [32]. F. Al-Zahrani et al. reported the development and analysis of a ring-core photonic crystal fiber with high refractive index separation, capable of supporting 76 OAM modes and six LP modes for large-speed high-range optical communication networks [33]. The free space transmission of OAM modes showing a spectral efficiency of $95.7 \mathrm{bit} / \mathrm{sec} / \mathrm{Hz}$ with a net information rate of $100.8 \mathrm{Tbps}$ and a $1.1 \mathrm{~km}$ MMF transmission of OAM modes to realize a 1.6 Tbps optical fiber network has been discussed by J. Wang et al. in reference [34].

The application of OAM modes to realize high-speed FSO links has also been reported by different research groups [35-39]. The designing and evaluation of a low-density parity check coded FSO link incorporating high-capacity transmission under strong turbulence conditions using OAM multiplexing has been discussed by $\mathrm{Z} \mathrm{Qu}$. et al. in reference [35]. The use of OAM multiplexing for deep space applications and multi-gigabit nearEarth optical networks has been reported by I. Djordjevic in reference [36]. Z. Qu. et al. reported a multi-gigabit capacity FSO link incorporating hybrid OAM multiplexing and WDM techniques [37]. Further, the link performance under strong turbulent conditions has been improved by deploying adaptive optics and channel coding techniques. L. Li et al. reported an OAM-multiplexed FSO communication system, where 80-Gbps information is transmitted between two ground terminals separated at $100 \mathrm{~m}$ via an unmanned aerial vehicle (UAV) using two independent 40-Gbps QPSK-modulated OAM beams [38]. Z. Zhao et al. reported an ultrahigh capacity FSO communication system over strong atmospheric turbulence conditions by incorporating hybridization of OAM multiplexing, polarization multiplexing, and frequency multiplexing [39]. The research works in references [40-44] report the simulative analysis of MDM-based high-capacity radio over fiber (RoF) links.

The main motivation here is to model an FSO link capable of securely transmitting biosensor data in health-care facilities under different atmospheric conditions at high bit rates. We discuss the simulation designing and evaluation of the OFDMFSO link with high-speed data transmission capabilities using WDM and MDM techniques under different atmospheric conditions. The link design is reported in Link Design of Wavelength-Mode Division Multiplexing-Orthogonal Frequency Division Multiplexing-Based Free Space Optics Link Section, and the simulative evaluation results are discussed in Numerical Results Section. Conclusion Section concludes this research work.

\section{LINK DESIGN OF WAVELENGTH-MODE DIVISION MULTIPLEXING-ORTHOGONAL FREQUENCY DIVISION MULTIPLEXING-BASED FREE SPACE OPTICS LINK}

Figure 1 presents the schematic design of the proposed FSO link. Optisystem $^{\mathrm{TM}}$ simulation software v.15 has been used for designing and evaluating the FSO link.

Four 20-Gbps OFDM-encoded data signals are transported over the FSO channel under different weather conditions. Two channels ( 1 and 2) are transmitted at $193.1-\mathrm{THz}$ frequency over HG01 and HG03 modes, and another two channels (3 and 4) are transmitted at $193.2 \mathrm{THz}$ frequency over HG01 and HG03 modes. A WDM multiplexer is used to combine the two frequencies at the transmitter side. Figure 2 presents the optical spectrum of the transmitted signal.

The HG modes can be mathematically described using the equation [45]:

$$
\begin{aligned}
\varphi_{r, s}(x, y)= & H_{m}\left(\frac{\sqrt{2} x}{\omega_{0, x}}\right) \exp \left(-\frac{x^{2}}{\omega_{o x}^{2}}\right) \exp \left(j \frac{\pi x^{2}}{\lambda R_{o x}}\right) \\
& \times H_{n}\left(\frac{\sqrt{2} y}{w_{o, y}}\right) \exp \left(-\frac{y^{2}}{w_{o y}^{2}}\right)\left(j \frac{\pi y^{2}}{\lambda R_{o y}}\right),
\end{aligned}
$$



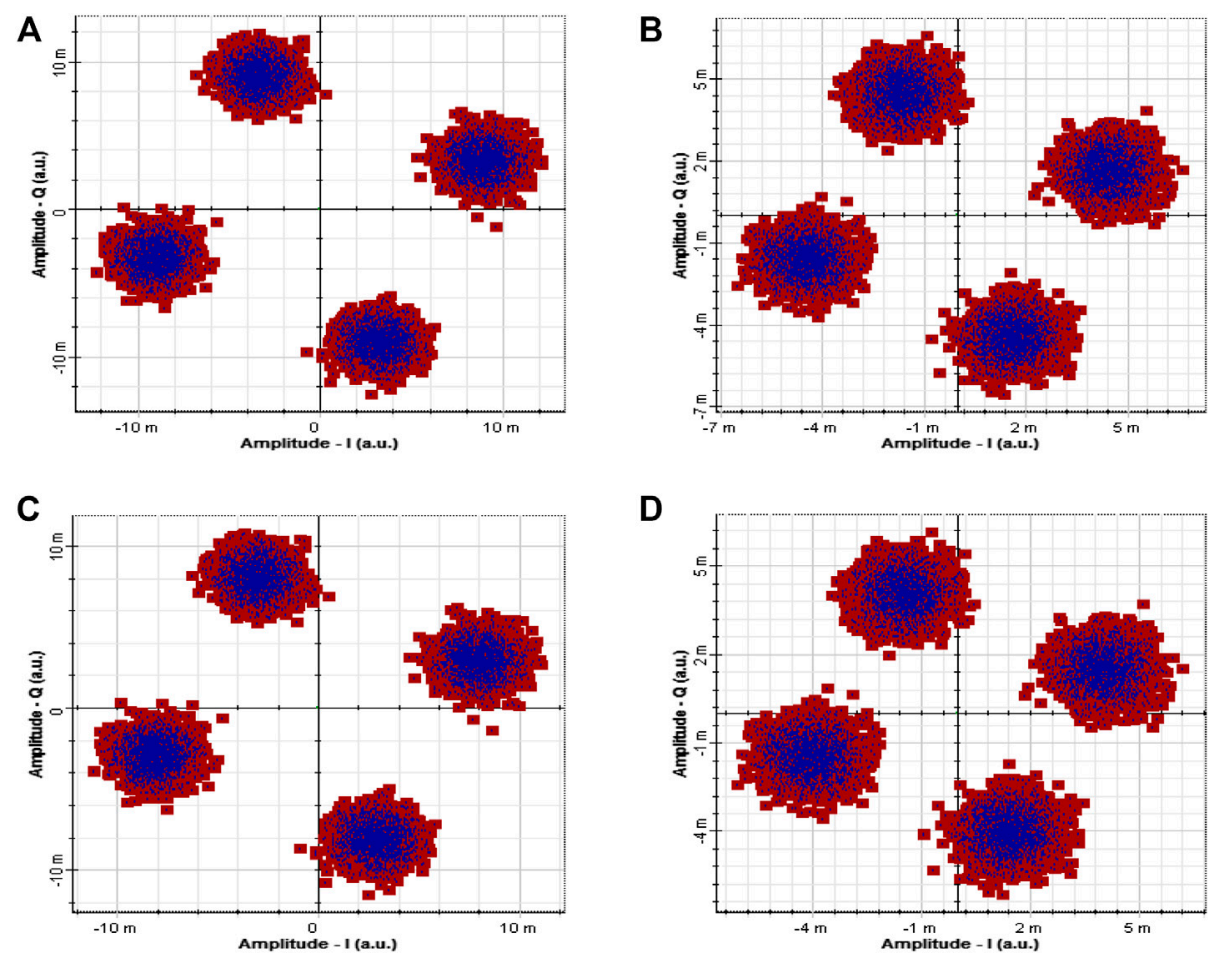

FIGURE 6 | Constellation plots after 32 km range for 193.1 THz channel (A) HG01 mode and (B) HG03 mode; for 193.2 THz channel (C) HG01 mode and (D) HG03 mode.
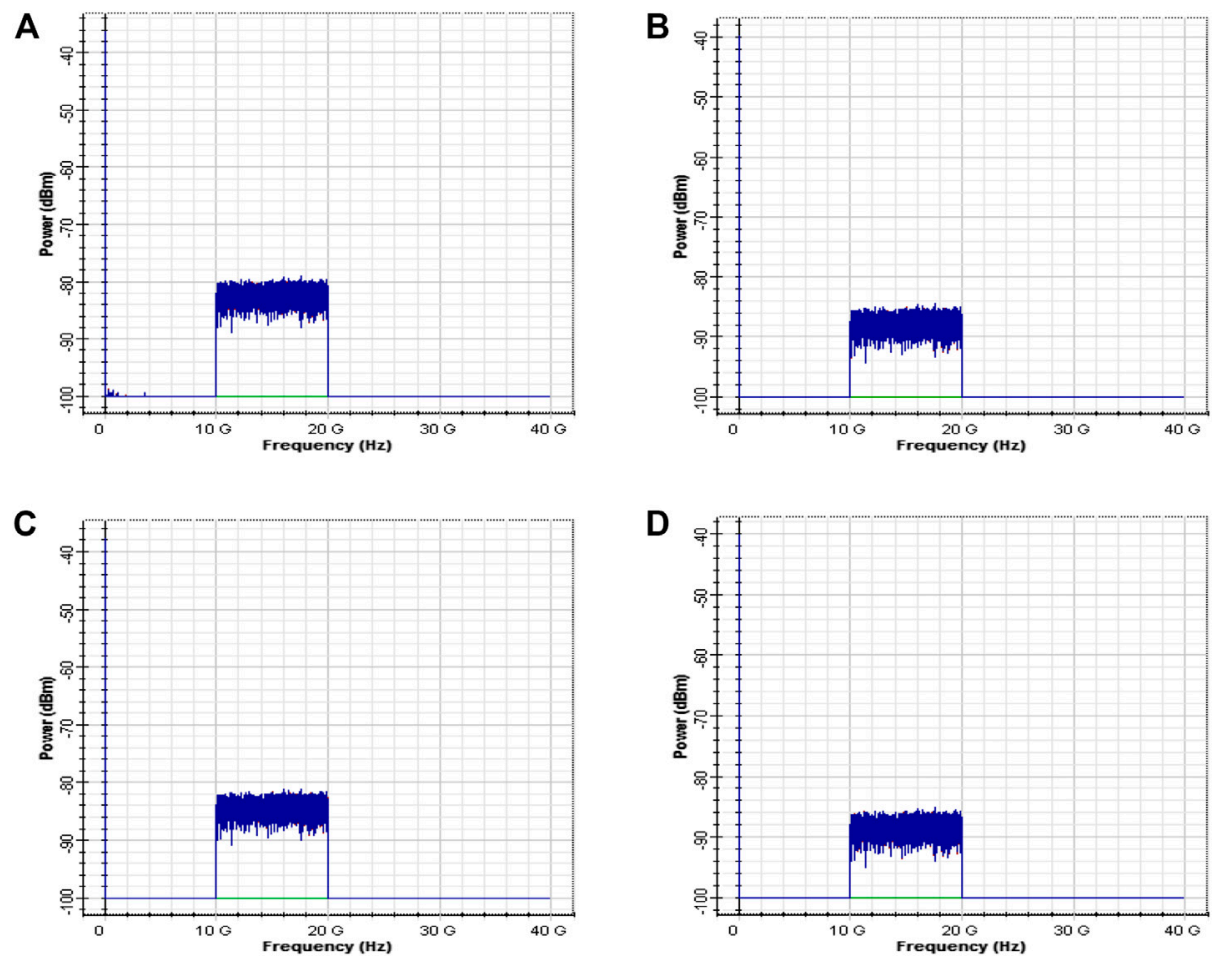

FIGURE 7|RF power after $32 \mathrm{~km}$ range for $193.1 \mathrm{THz}$ channel (A) HG01 mode and (B) HG03 mode; for 193.2 THz channel (C) HG01 mode and (D) HG03 mode. 

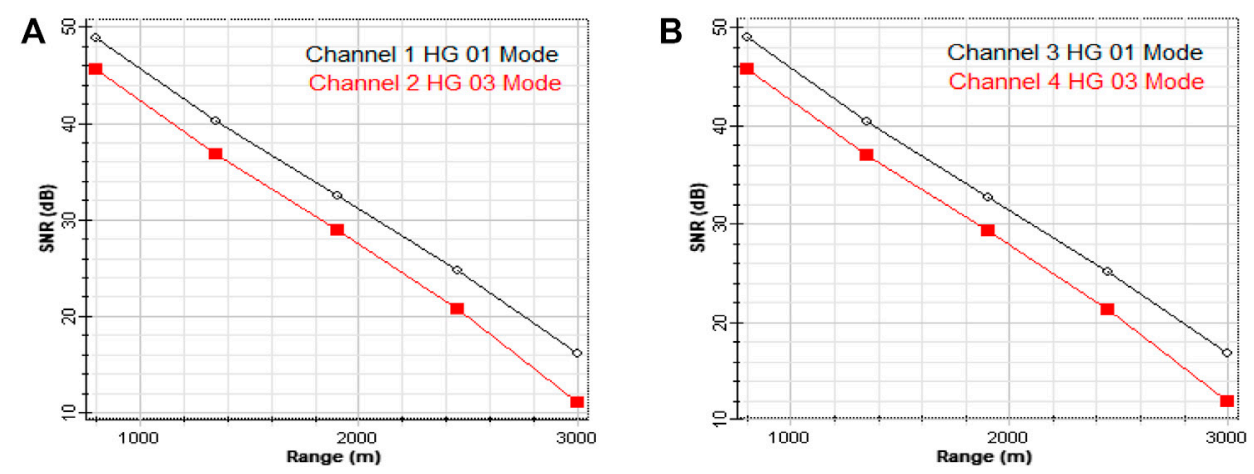

FIGURE 8 | SNR plots for (A) 1931. THz channel and (B) 193.2 THz channel under low fog.
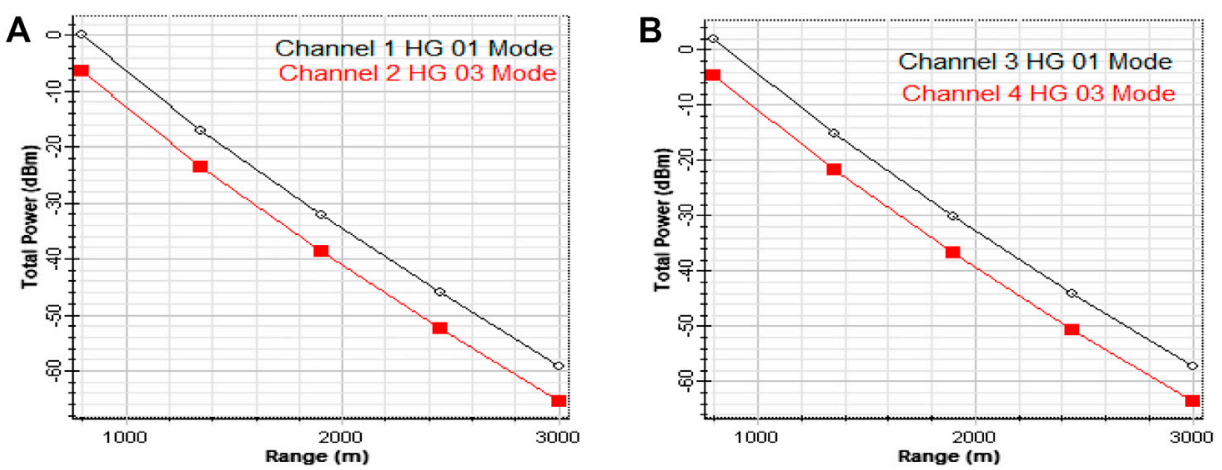

FIGURE 9 | Signal power plots for (A) 1931. THz channel and (B) 193.2 THz channel under low fog.
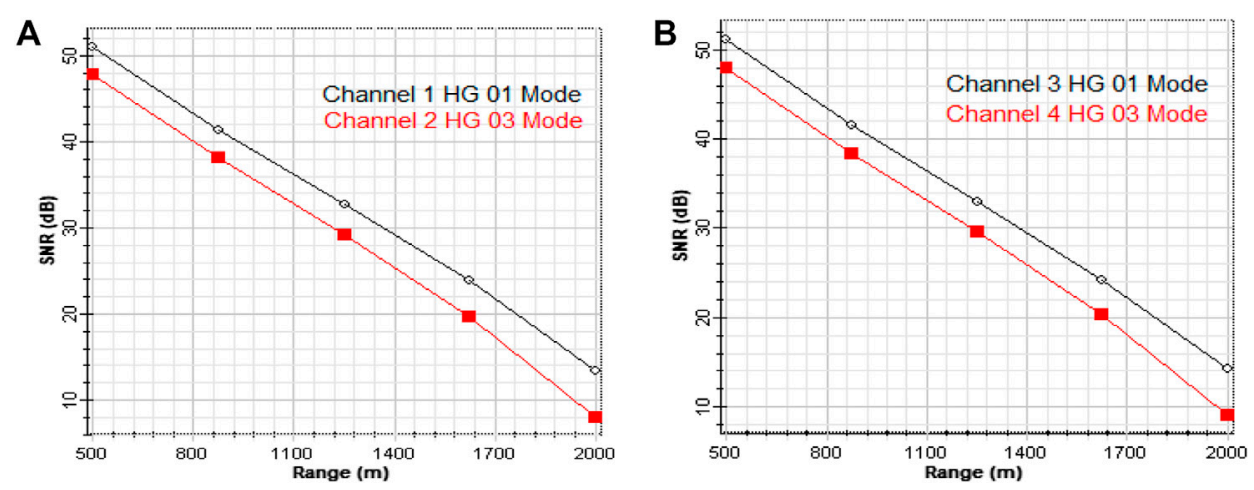

FIGURE 10 | SNR plots for (A) 1931. THz channel and (B) 193.2 THz channel under heavy fog.

where $r$ is the dependency of mode profile on the $X$-axis, $s$ is the dependency of mode profile on the $Y$-axis, the radius of the beam is denoted by $R$, the size of the optical beam at the waist is denoted by $\omega_{0}$, and $H_{m}$ and $H_{n}$ denote Hermite polynomials. Different HG modes are excited using a spatial laser, and the mode intensity profiles are illustrated in Figure 3.

For each channel, 20-Gbps data from the information source is mapped onto 4-QAM symbols, where two bits are transmitted per symbol. This signal is further OFDM modulated in the electrical domain. The specification of the OFDM modulator is 1,024 inverse fast Fourier transformation points, 512 orthogonal subcarriers, a cyclic prefix of value 32, and average power of $15 \mathrm{dBm}$. This signal is up-converted using a $7.5-\mathrm{GHz}$ quadrature amplitude (QM) modulator. For each frequency channel, the 4-QAM-OFDM spatially modulated signals are combined using an MDM multiplexer (MUX). The distinct frequency channels are then 

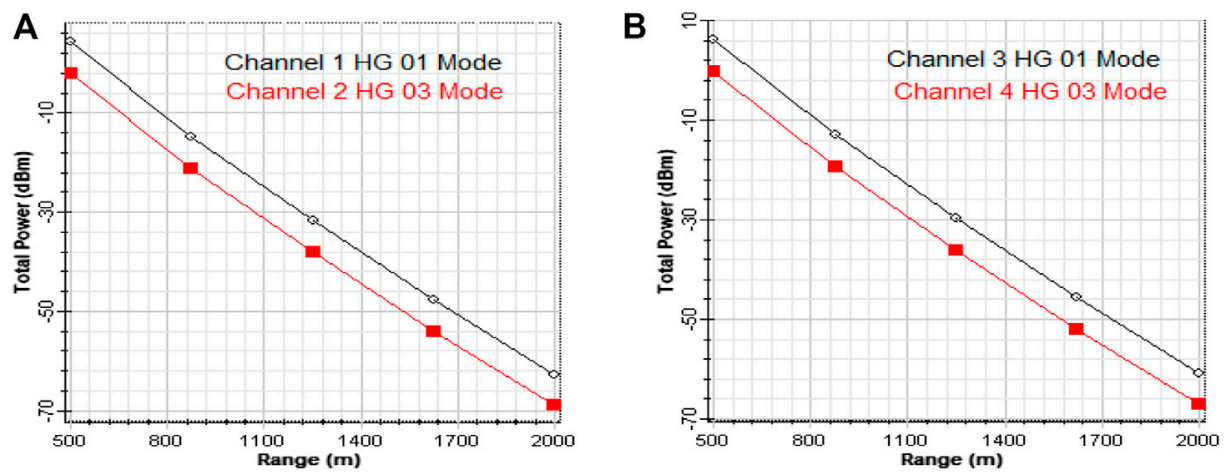

FIGURE 11 | Signal power plots for (A) 1931. THz channel and (B) $193.2 \mathrm{THz}$ channel under heavy fog.
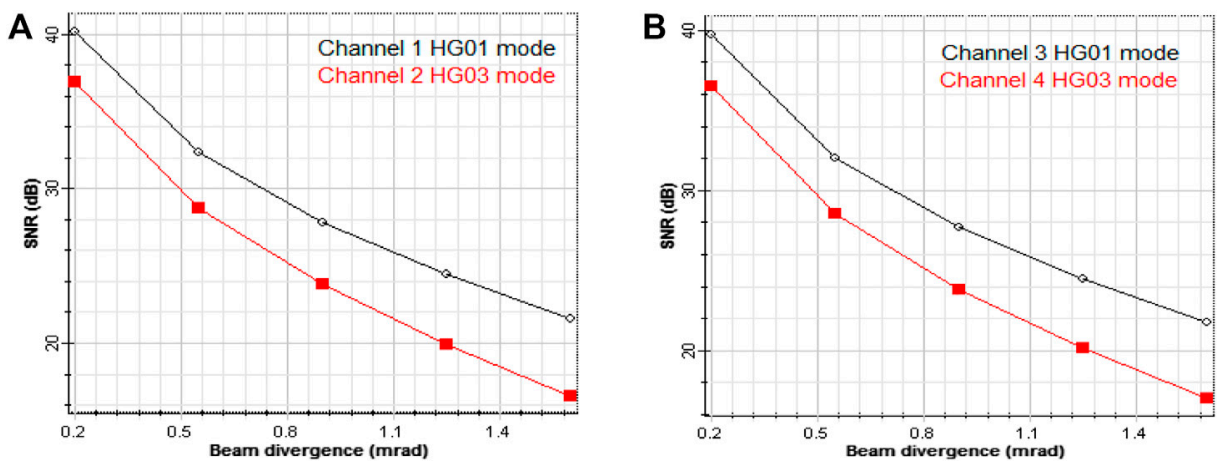

FIGURE 12 | SNR plots for (A) 1931. THz channel and (B) 193.2 THz channel under the effect of the beam divergence angle.
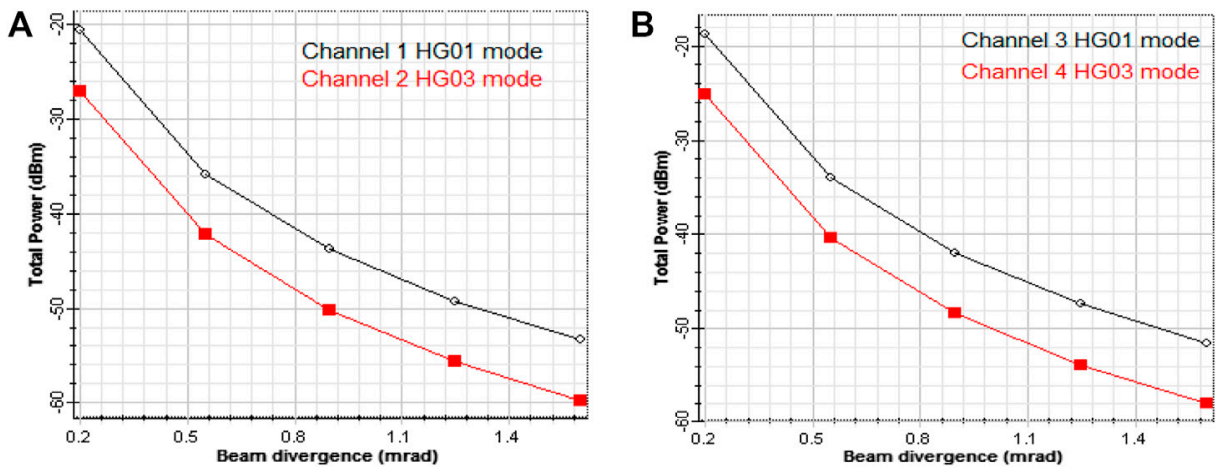

FIGURE 13 | Signal power plots for (A) 1931. THz channel and (B) $193.2 \mathrm{THz}$ channel under the effect of the beam divergence angle.

multiplexed using a WDM MUX (Figure 2), and the information signal is transmitted using a transmitter lens.

The link equation can be described as [46]:

$$
P_{\text {Received }}=P_{\text {Transmitted }}\left(\frac{d_{R}^{2}}{\left(d_{T}+\theta Z\right)^{2}}\right) 10^{-\sigma Z / 10},
$$

where $d_{R}$ denotes the aperture diameter of receiver lens $(100 \mathrm{~mm}), d_{T}$ denotes aperture diameter of transmitter lens
( $100 \mathrm{~mm}), \theta$ denotes the size of the optical beam/divergence angle $(0.25 \mathrm{mrad}), \mathrm{Z}$ denotes the FSO range, and $\sigma$ is the attenuation coefficient for varying climate conditions. The attenuation coefficient for low fog, heavy fog, and clear conditions is 9,22 , and $0.14 \mathrm{~dB} / \mathrm{km}$, respectively [47]. At the receiver, individual frequency channels are separated using the WDM de-multiplexer (DEMUX), and for each frequency channel, independent spatial channels are separated using 
MDM DEMUX. An APD photodiode converts the optical signal into its electrical equivalent. Originally transmitted message bits are recovered using OFDM and QM demodulator sections.

\section{NUMERICAL RESULTS}

Figures 4, 5 illustrate plots for SNR and the signal power with an increasing range in the proposed link under clear weather. Figure $\mathbf{4 A}$ shows that channel 1 transmitted over the HG01 mode performs notably better than channel 2 transmitted over the HG03 mode at $193.1 \mathrm{THz}$ frequency. For channel 1, the SNR value at the receiver terminal is measured as $34.67,23.75$, and $15.44 \mathrm{~dB}$, whereas for channel 2, SNR is reported as $31.29,19.62$, and $10.31 \mathrm{~dB}$ at 10, 25, and $40 \mathrm{~km}$, respectively. Figure $4 \mathrm{~B}$ shows that for channel 3, the SNR value is $34.88,24.14$, and $16.16 \mathrm{~dB}$, whereas for channel 4 , the SNR value is $31.52,20.16$, and $11.23 \mathrm{~dB}$ at 10,25 , and $40 \mathrm{~km}$, respectively. It can be seen that for the 193.2 THz frequency channel, the HG01 mode outperforms the HG03 mode.

Figure 5A shows that for the channel 1 , the signal power is $-27.93,-47.78$, and $-60.02 \mathrm{dBm}$, whereas for channel 2 , the signal power is $-34.39,-54.22$, and $-66.32 \mathrm{dBm}$ at 10,25 , and $40 \mathrm{~km}$, respectively. Figure 5B shows that for channel 3, the signal power is $-26.03,-45.88$, and $-58.14 \mathrm{dBm}$, whereas for channel 4 , the signal power is $-32.49,-52.33$, and $-64.49 \mathrm{dBm}$ at 10,25 , and $40 \mathrm{~km}$, respectively. A feasible transmission of $4 \times$ $20 \mathrm{Gbps}$ information at $32 \mathrm{~km}$ with fair performance metrics (SNR 20 dB) [40] is observed from the results presented. Figure 6 reports constellation plots, and Figure 7 reports the $\mathrm{RF}$ power of the signals at $32 \mathrm{~km}$.

Further, the W-MDM-OFDM-based FSO link is evaluated for low and heavy fog conditions. Figures 8, 9 report SNR and signal power plots for different channels under low fog conditions in the proposed link. Figures 8A,B show that the SNR reduces from $48.83,45.59,49.01$, and $45.77 \mathrm{~dB}$ to $16.13,11.07,16.82$, and $11.96 \mathrm{~dB}$ for channels $1,2,3$, and 4 , respectively, for the link range increasing from 800 to $3,000 \mathrm{~m}$ under low fog conditions. Similarly, Figure 9A,B show that the signal power reduces from $0.04,-6.41,1.94$, and $-4.51 \mathrm{dBm}$ to $-59.12,-65.44,-57.23$, and $-63.60 \mathrm{dBm}$ for channels $1,2,3$, and 4 , respectively, for the link range increasing from 800 to $3,000 \mathrm{~m}$ under low fog conditions.

Figures 10, 11 present SNR and signal power plots for different channels under heavy fog conditions. Figures 10A,B show that the SNR reduces from $50.96,47.72,51.14$, and $47.90 \mathrm{~dB}$ to $13.36,8.01,14.17$, and $9.01 \mathrm{~dB}$ for channels $1,2,3$, and 4 , respectively, for the link range increasing from 500 to $2000 \mathrm{~m}$ under heavy fog conditions. Similarly, Figures 11A,B show that the signal power reduces from $4.29,-2.16,6.18$, and $-0.26 \mathrm{dBm}$ to $-62.67,-68.87,-60.81$, and $-67.09 \mathrm{dBm}$ for channels $1,2,3$, and 4 , respectively, for the link range increasing from 500 to $2000 \mathrm{~m}$ under heavy fog conditions. It can be observed that for low fog, the link prolongs to 2,800 $\mathrm{m}$, whereas for heavy fog, $1750 \mathrm{~m}$ range is supported with fair performance (SNR $20 \mathrm{~dB}$ ).

In this study, we also discuss the impact of the increasing beam divergence angle on the performance of the proposed link. Figures 12, 13 demonstrate SNR and signal power plots, respectively, with an increasing angle of beam divergence. From the results presented, it can be seen that SNR varies from 39.73, $36.85,40.12$, and $36.49 \mathrm{~dB}$ to $21.57,17.10,21.75$, and $16.57 \mathrm{~dB}$ as the beam divergence angle increases from 0.2 to $1.6 \mathrm{mrad}$ for channels 1 , 2,3 , and 4 respectively. Alternatively, the signal power reduces from $-20.59,-27.05,-18.73$, and $-25.19 \mathrm{dBm}$ to $-53.35,-59.76,-51.50$, and $-57.92 \mathrm{dBm}$ as the beam divergence angle increases from 0.2 to 1.6 mrad for channels $1,2,3$, and 4, respectively. Degradation in the received signal quality with the increasing angle of beam divergence can be observed from the reported results. This is because increasing beam size results in lesser optical power collected at the receiver plane and higher power lost to the surroundings, thus degradation in the link performance.

\section{CONCLUSION}

In this study, an FSO link is proposed for providing biomedical services, and we report a successful transmission of $4 \times 20$-Gbps data over an OFDM-based FSO link by incorporating hybrid WDM and MDM techniques under different atmospheric conditions. From the results presented, it can be concluded that the proposed link prolongs to $32 \mathrm{~km}$ with acceptable performance (SNR 20 dB) under clear weather conditions which reduces to $2,800 \mathrm{~m}$ and $1750 \mathrm{~m}$ under low fog and heavy fog conditions, respectively. Also, the HG01 mode performs better than the HG03 mode since the former has more immunity against fading effects due to adverse weather conditions. Also, the performance of the proposed link under an increasing beam divergence angle has been discussed. From the results presented, it can be observed that the performance of the proposed link degrades in terms of SNR and signal power of the received signal on increasing the beam divergence angle. In future studies, dual-polarization transmission along with digital signal processing techniques at the receiver side can be incorporated in the proposed system to further enhance the information capacity and link performance under adverse climate conditions.

\section{DATA AVAILABILITY STATEMENT}

The original contributions presented in the study are included in the article/Supplementary Material; further inquiries can be directed to the corresponding author.

\section{AUTHOR CONTRIBUTIONS}

All authors listed have made a substantial, direct, and intellectual contribution to the work and approved it for publication.

\section{ACKNOWLEDGMENTS}

The researchers would like to thank the Deanship of Scientific Research, Qassim University for funding the publication of this project. 


\section{REFERENCES}

1. Khalighi MA, and Uysal M. Survey on Free Space Optical Communication: A Communication Theory Perspective. IEEE Commun Surv Tutorials (2014) 16(4):2231-58. doi:10.1109/comst.2014.2329501

2. Chaudhary S, Sharma A, Tang X, Wei X, and Sood P. A Cost Effective 100 Gbps FSO System under the Impact of Fog by Incorporating OCDMA-PDM Scheme. Wireless Pers Commun (2021) 116:2159-68. doi:10.1007/s11277-02007784-3

3. Nykolak G, Szajowski PF, Tourgee G, and Presby H. 2.5 Gbit/s Free Space Optical Link over 4.4 Km. Electron Lett (1999) 35(7):578-9. doi:10.1049/el: 19990377

4. Singh M, and Malhotra J. $4 \times 20 \mathrm{Gbit} / \mathrm{s}-40 \mathrm{GHzOFDM}$ Based Radio over FSO Transmission Link Incorporating Hybrid Wavelength Division MultiplexingMode Division Multiplexing of LG and HG Modes with Enhanced Detection. Optoelectronics Adv Mater - Rapid Commun (2020) 14:5233-6243.

5. Ramezani A, Noroozi MR, and Aghababaee M. Analyzing Free Space Optical Communication Performance. Int J Eng Adv Technol (2014) 4(1):46-51.

6. Singh J, and Kumar N. Performance Analysis of Different Modulation Format on Free Space Optical Communication System. Optik - Int J Light Electron Opt (2013) 124(20):4651-4. doi:10.1016/j.ijleo.2013.02.014

7. Saruchi Attri S, Charu Narula C, and Sanjiv Kumar S. Performance Analysis of FSO Link Using CO-OFDM under the Effect of Atmospheric Turbulence. In: Proc. Of International Conference on Intelligent Communication, Control, Devices. Dehradun, India: Springer (2017). p. 167-72. doi:10.1007/978-98110-1708-7_18

8. Amphawan A, Chaudhary S, and Chan VWS. 2 X 20 Gbps - $40 \mathrm{GHz}$ OFDM Ro-FSO Transmission with Mode Division Multiplexing. J Eur Opt Soc - Rapid publications (2014) 9:14041. doi:10.2971/jeos.2014.14041

9. Cvijetic N, Qian D, and Wang T. 10 GB/s Free-Space Optical Transmission Using OFDM. In: OFC/NFOEC; 24-28 February 2008; San Diego, CA, USA (2007). p. 1-3.

10. Gill HK, Dhaliwal BS, and Singh K. Performance of OFDM FSO System Using ODSB, OSSB, and OVSB Modulation Schemes by Deploying Spatial Diversity. Int J Eng Develop Res (2015) 3(2):1384-9.

11. Chaudhary S, Tang X, Ghassemlooy Z, Lin B, Wei X, and Liaw KS. A $3 \times 25$ Mbps WDM-Ro-VLC System for Amateur Radio Applications. In: 2019 2nd West Asian Colloquium on Optical Wireless Communications (WACOWC); 27-28 April 2019; Tehran, Iran (2019). p. 6-10. doi:10.1109/ WACOWC.2019.8770208

12. Sharma A, Kaur S, and Chaudhary S. Performance Analysis of 320 Gbps DWDM-FSO System under the Effect of Different Atmospheric Conditions. Opt Quant Electron (2021) 53:239. doi:10.1007/s11082-021-02904-0

13. Chaudhary S, Chauhan P, and Sharma A. High Speed $4 \times 2.5 \mathrm{Gbps}-5 \mathrm{GHz}$ AMI-WDM-RoF Transmission System for WLANs. J Opt Commun (2019) 40(3):285-8. doi:10.1515/joc-2017-0082

14. Chaudhary S, Chaudhary N, Sharma S, and Choudhary B. High Speed InterSatellite Communication System by Incorporating Hybrid PolarizationWavelength Division Multiplexing Scheme. J Opt Commun (2017) 39(1): 87-92. doi:10.1515/joc-2016-0107

15. Randel S, Ryf R, Sierra A, Winzer PJ, Gnauck AH, bolle CA, Essiambre RJ, et al. Space-division Multiplexing over $10 \mathrm{Km}$ of Three-Mode Fiber Using Coherent 6_ 6 MIMO Processing. In: proceedings to Optical Fiber Communication Conference and Exposition (OFC/NFOEC), 2011 and the National Fiber Optic Engineers Conference; 6-10 March 2011; Los Angeles. IEEE (2011). p. 1-3.

16. Amphawan A, and Dominic O. Modal Decomposition of Output Field for Holographic Mode Field Generation in a Multimode Fiber Channel. In: proceedings to Photonics (ICP), 2010 International Conference; 5-7 July 2010; Langkawi. IEEE (2010). doi:10.1109/icp.2010.5604377

17. Amphawan A, Mishra V, Nisar K, and Nedniyom B. Real-time Holographic Backlighting Positioning Sensor for Enhanced Power Coupling Efficiency into Selective Launches in Multimode Fiber. J Mod Opt (2012) 59:1745-52. doi:10.1080/09500340.2012.739713

18. Amphawan A. Binary Encoded Computer Generated Holograms for Temporal Phase Shifting. Opt Express (2011) 19:23085-96. doi:10.1364/oe.19.023085

19. Amphawan A. Binary Spatial Amplitude Modulation of Continuous Transverse Modal Electric Field Using a Single Lens for Mode Selectivity in
Multimode Fiber. J Mod Opt (2012) 59:460-9. doi:10.1080/ 09500340.2011.636486

20. Jung Y, Chen R, Ismaeel R, Brambilla G, Alam S-U, Giles IP, et al. Dual Mode Fused Optical Fiber Couplers Suitable for Mode Division Multiplexed Transmission. Opt Express (2013) 21:24326-31. doi:10.1364/oe.21.024326

21. Amphawan A, Benjaporn N, and Nashwan MAS. Selective Excitation of LP01 Mode in Multimode Fiber Using Solid-Core Photonic crystal Fiber. J Mod Optic (2014) 60:1-9. doi:10.1080/09500340.2013.827249

22. Juarez AA, Warm S, Bunge C-A, Krummrich PM, and Petermann K. Perspectives of Principal Mode Transmission in a Multi-Mode Fiber. In: 36th European Conference and Exhibition on Optical Communication; 1923 Sept. 2010; Torino (2010). p. 1-3. doi:10.1109/ECOC.2010.5621235

23. Kodama T, Isoda T, Morita K, Maruta A, Maruyama R, Kuwaki N, et al. First Demonstration of a Scalable MDM/CDM Optical Access System. Opt Express (2014) 22:12060-9. doi:10.1364/oe.22.012060

24. Masunda T, and Amphawan A. Feedforward Equalizers for MDM-WDM in Multimode Fiber Interconnects. J Opt Commun (2018) 39(2):147-53. doi:10.1515/joc-2016-0037

25. Murad R, Amphawan A, Fazea Y, Sajat M, and Alias H. MDM of Hybrid Modes in Multimode Fiber. In: Proceeding of International Conference on Electrical Engineering, Computer Science and Informatics, Indonesia; 19-20 August 2015; alembang, Indonesia (2015). p. 117-22.

26. Hamed EK, Munshid MA, and Hmood JK. Effect of Few-Mode Fiber Profile on Long-Haul MDM Transmission. AIP Conf Proc (2020) 2213(1):020137. doi:10.1063/5.0000088

27. Feng Z, Xu L, Wu Q, Tang M, Fu S, Tong W, et al. Ultra-high Capacity WDM-SDM Optical Access Network with Self-Homodyne Detection Downstream and 32QAMFBMC Upstream. Opt Express (2017) 25(6):5951-61. doi:10.1364/OE.25.005951

28. Brunet C, Ung B, Messaddeq Y, LaRochelle S, Bernier E, and Rusch LA. Design of an Optical Fiber Supporting 16 OAM Modes. In: OFC 2014; 9-13 March 2014; San Francisco, CA (2014). p. 1-3. doi:10.1364/OFC.2014.Th2A.24

29. Zhang $X$, Zhang H, and Li H. The OAM Transmission Fiber Based on Circular Photonic crystal Fiber Structure. In: 2017 16th International Conference on Optical Communications and Networks (ICOCN); 7-10 Aug. 2017; Wuzhen, China (2017). p. 1-3. doi:10.1109/ICOCN.2017.8121421

30. Hassan MM, Kabir MA, Hossain MN, Biswas B, Paul BK, and Ahmed K. Photonic crystal Fiber for Robust Orbital Angular Momentum Transmission: Design and Investigation. Opt Quant Electron (2020) 52:8. doi:10.1007/s11082-019-2125-0

31. Ingerslev K, Gregg P, Galili M, Da Ros F, Hu H, Bao F, et al. 12 Mode, WDM, MIMO-free Orbital Angular Momentum Transmission. Opt Express (2018) 26: 20225-32. doi:10.1364/OE.26.020225

32. Tatarczak A, Usuga AM, and Monroy IT. OAM-enhanced Transmission for Multimode Short-Range Links. In: Proc. SPIE 9390, Next-Generation Optical Networks for Data Centers and Short-Reach Links II; 9 March 2015; San Francisco, California, United States (2015). p. 93900E. doi:10.1117/12.2079795

33. Al-Zahrani FA, and Ahmed K. Novel Design of Dual Guided Photonic crystal Fiber for Large Capacity Transmission in High-Speed Optics Communications with Supporting Good Quality OAM and LP Modes. Alexandria Eng J (2020) 59:4889-99. doi:10.1016/j.aej.2020.09.004

34. Wang J, and Willner AE. Using Orbital Angular Momentum Modes for Optical Transmission. In: Optical Fiber Communication Conference; 9-13 March 2014; San Francisco, CA, USA. San Francisco: Optical Society of America (2014). OSA Technical Digest (online) paper W4J.5. doi:10.1364/ ofc.2014.w4j.5

35. Qu Z, and Djordjevic IB. LDPC-coded OAM Based FSO Transmission System in the Presence of strong Atmospheric Turbulence. In: 2015 49th Asilomar Conference on Signals, Systems and Computers; 8-11 Nov. 2015; Pacific Grove, CA (2015). p. 999-1002. doi:10.1109/ACSSC.2015.7421288

36. Djordjevic IB. Deep-space and Near-Earth Optical Communications by Coded Orbital Angular Momentum (OAM) Modulation. Opt Express (2011) 19: 14277-89. doi:10.1364/OE.19.014277

37. Qu Z, and Djordjevic IB. Orbital Angular Momentum Multiplexed Free-Space Optical Communication Systems Based on Coded Modulation. Appl Sci (2018) 8:2179. doi:10.3390/app8112179

38. Li L, Zhang R, Zhao Z, Xie G, Liao P, Pang K, et al. High-Capacity Free-Space Optical Communications between a Ground Transmitter and a Ground Receiver via a UAV Using Multiplexing of Multiple Orbital-AngularMomentum Beams. Sci Rep (2017) 7:17427. doi:10.1038/s41598-017-17580-y 
39. Zhao L, Liu H, Hao Y, Sun H, and Wei Z. Effects of Atmospheric Turbulence on OAM-POL-FDM Hybrid Multiplexing Communication System. Appl Sci (2019) 9:5063. doi:10.3390/app9235063

40. Amphawan A, Chaudhary S, and Gupta B. Secure MDM-OFDM-Ro-FSO System Using HG Modes. Int J sensors, wireless Commun Control (2015) 5: 13-8. doi:10.2174/2210327905999150521110316

41. Sharma A, Chaudhary S, Thakur D, and Dhasratan V. A Cost Effective High Speed Radio over Fiber System for Millimeter Wave Applications. J Opt Commun (2017) 41:177-80. doi:10.1515/jjoc-2017-0166

42. Almogahed A, Amphawan A, and Fazea Y. Mitigation of Atmospheric Turbulences Using Mode Division Multiplexing Based on Decision Feedback Equalizer for Free Space Optics. J Opt Commun (2017) 41: 185-93. doi:10.1515/joc-2017-0169

43. Amphawan A, Fazea Y, Murad R, Alisa H, and Sajat M. MDM of Hybrid Modes in Multimode Fiber, Electrical Engineering. In: Computer Science and Informatics (EECSI); August 2015; Palembang, Indonesia (2015).

44. Fazea Y, and Amphawan A. 25 Gbit/s WDM-MDM. J Opt Commun (2016) 36: 327-33. doi:10.1515/joc-2014-0091

45. Ghatak A, and Thyagarajan K. An Introduction to Fiber Optics. Cambridge: Cambridge University Press (1998).

46. Kolev DR, Wakamori K, and Matsumoto M. Transmission Analysis of OFDM-Based Services over Line-Of-Sight Indoor Infrared Laser Wireless
Links. J Lightwave Technol (2012) 30:3727-2735. doi:10.1109/ jlt.2012.2227456

47. Sarangal H, Singh A, Malhotra J, and Chaudhary S. A Cost Effective 100 Gbps Hybrid MDM-OCDMA-FSO Transmission System under Atmospheric Turbulences. Opt Quant Electron (2017) 49:184. doi:10.1007/s11082-017-1019-2

Conflict of Interest: The authors declare that the research was conducted in the absence of any commercial or financial relationships that could be construed as a potential conflict of interest.

Publisher's Note: All claims expressed in this article are solely those of the authors and do not necessarily represent those of their affiliated organizations, or those of the publisher, the editors, and the reviewers. Any product that may be evaluated in this article, or claim that may be made by its manufacturer, is not guaranteed or endorsed by the publisher.

Copyright (c) 2021 Singh, Chebaane, Ben Khalifa, Grover, Dewra and Angurala. This is an open-access article distributed under the terms of the Creative Commons Attribution License (CC BY). The use, distribution or reproduction in other forums is permitted, provided the original author(s) and the copyright owner(s) are credited and that the original publication in this journal is cited, in accordance with accepted academic practice. No use, distribution or reproduction is permitted which does not comply with these terms. 